\title{
PERFIL DE CRIANÇAS DEPENDENTES DE TECNOLOGIA NO MUNICIPIO DE RIBEIRÃO PRETO-SP
}

\author{
PROFILE OF TECHNOLOGY-DEPENDENT CHILDREN IN \\ RIBEIRÃO PRETO-SP
}

\author{
Aline Cristiane Cavicchioli Okido ${ }^{1}$, Miyeko Hayashida ${ }^{2}$, Regina Aparecida Garcia Lima ${ }^{3}$
}

\begin{abstract}
Resumo:
Estudo descritivo, exploratório, com abordagem quantitativa que objetivou caracterizar crianças dependentes de tecnologia, residentes em Ribeirão Preto - SP, com relação às condições de gestação e nascimento, origem das necessidades especiais de saúde e demandas de cuidados. Participaram 102 mães ou responsáveis. Os dados foram obtidos no período de janeiro a abril de 2011 , mediante aplicação de formulário, durante visita domiciliar. Os resultados revelaram que $57 \%$ das crianças são do sexo masculino; a faixa etária predominante foi de 1 a 4 anos; $7,8 \%$ das mães não fizeram acompanhamento pré-natal; $96 \%$ nasceram em instituições hospitalares; $63,7 \%$ de parto cesárea e a prematuridade ocorreu em $29,3 \%$ dos casos. Quanto à origem das necessidades especiais de saúde, $65,7 \%$ possuíam algum problema congênito, 30,4\% tiveram intercorrências durante o parto e ou no período neonatal e 30,4\% apresentaram intercorrências ao longo da vida, definidas como causas adquiridas. Quanto às demandas de cuidados especiais, todas apresentam demanda de cuidado tecnológico, 92 necessitam de cuidado medicamentoso, 74 requerem cuidado de desenvolvimento e 102 cuidados habituais modificados. 0 estudo aponta dados relevantes para ampliar a visibilidade dessa clientela nas taxas oficiais e nas políticas públicas de forma a assegurar uma assistência qualificada e integral.
\end{abstract}

Palavras-chave: enfermagem Pediátrica; cuidado da criança; doença crônica.

\begin{abstract}
:
The aim of this descriptive and exploratory research with a quantitative approach was to characterize technology-dependent children living in Ribeirão Preto - SP regarding gestation and birth conditions, origin of the special health needs and care demands. Participants were 102 mothers or responsible caregivers. Data were collected between January and April 2011 through the application of a form during home visits. The results revealed that $57 \%$ of the children are male; the predominant age range was between 1 and 4 years; $7.8 \%$ of the mothers had no prenatal follow-up; $96 \%$ were born in hospitals; $63.7 \%$ through c-section and, in $29.3 \%$ of cases, the children were premature. As for the origin of the special health needs, $65.7 \%$ had some congenital problem, $30.4 \%$ experienced problems during delivery and/or in the neonatal period and $30.4 \%$ experienced problems during the lifetime, defined as acquired causes. Regarding special care demands, all children display technological care demands, 92 need medication care, 74 developmental care and 102 modified habitual care. The study appoints relevant data to broaden these clients' visibility in official rates and public policies, so as to guarantee qualified and comprehensive care delivery.
\end{abstract}

Key words: pediatric nursing; child care; chronic Illness.

1 Enfermeira. Doutoranda do Programa de Pós-Graduação Enfermagem em Saúde Publica da Escola de Enfermagem de Ribeirão Preto da Universidade de São Paulo.

2 Enfermeira. Doutora em Enfermagem pela Escola de Enfermagem de Ribeirão Preto da Universidade de São Paulo.

3 Enfermeira. Orientadora. Professor Titular do Departamento de Enfermagem Materno Infantil e Saúde Pública da Escola de Enfermagem de Ribeirão Preto da Universidade de São Paulo.

Corresponding author: acc@eerp.usp.br

Suggested citation: Okido ACC, Hayashida M, Lima RAG. Profile of technology-dependent children in Ribeirão Preto-SP. J. Hum. Growth Dev. 2012; 22(3): 291-296

Manuscript submitted Sep 18 2011, accepted for publication Mar 302012 


\section{INTRODUÇÃO}

O perfil epidemiológico das crianças, no Brasil, tem-se modificado. Num primeiro momento, as doenças imunopreveníveis contribuíram expressivamente para o quadro de morbimortalidade e, atualmente, as afecções perinatais desenham uma nova tendência neste cenário, sendo os óbitos neonatais precoces responsáveis por mais da metade dos óbitos de crianças brasileiras no primeiro ano de vida, indicando a necessidade de adequação dos processos assistenciais que vão desde o atendimento individual até o processo de organização da rede de assistência à gestante, ao parto e ao recém nascido ${ }^{1-3}$. Em adição, outros fatores contribuíram para essa mudança, tais como a melhoria das condições ambientais e nutricionais da população infantil brasileira, a implementação de programas, estratégias e ações de saúde direcionadas ao quadro de morbimortalidade infantil, como, por exemplo, o Programa Nacional de Imunização (PNI) e, por fim, a incorporação de novas tecnologias na recuperação das doenças infantis por causas congênitas e adquiridas ${ }^{1}$.

Nessa perspectiva, com os avanços das políticas públicas e da prática médica no cuidado às crianças pré-termo, com anomalias congênitas e doenças crônicas, emergiu um grupo de crianças que necessita de suporte tecnológico para manutenção de suas vidas ${ }^{4}$, sendo estas integrantes do grupo conhecido como crianças com necessidades especiais de saúde (CRIANES) ${ }^{5}$, as quais, na literatura internacional, são denominadas de Children with special healthcare needs ${ }^{6}$.

As CRIANES são crianças que requerem cuidados individuais e personalizados, apresentam saúde debilitada e necessitam de acompanhamento dos serviços de saúde e sociais para além do necessário a uma criança saudável|4-6. Seus comprometimentos podem ser físicos, de desenvolvimento e de comportamento, necessitando, algumas vezes, do uso de tecnologias mais sofisticadas que garantam sua sobrevivência; neste caso, as crianças dependentes de tecnologia. Em estudo realizado com crianças do Reino Unido7, foram encontrados os seguintes tipos de artefatos ou dispositivos: traqueostomia, cateter nasal, torpedo de oxigênio, concentrador de oxigênio, ventilação mecânica, drogas intravenosas, nutrição parenteral, diálise peritoneal e gastrostomia.

Outra classificação para as crianças com necessidade especiais de saúde relaciona-se à demanda de cuidado exigido: aquelas com demanda de cuidado de desenvolvimento, ou seja, crianças com disfunções neuromotoras; as que demandam cuidado medicamentoso; as que demandam cuidados habituais modificados, compreendendo os cuidados especiais do dia a dia que vão além daquele oferecido a uma criança considerada saudável, por exemplo, monitorização dos sinais vitais e saturação de oxigênio e, por fim, aquelas que demandam cuidado tecnológico, integrando o grupo de depen- dentes, do qual fazem parte crianças dependentes de algum dispositivo para manutenção da vida. É importante ressaltar que essa classificação é meramente didática, pois uma criança pode requerer mais de uma demanda de cuidado ${ }^{8}$.

Estudos $^{9-10}$ identificam ausência de registros do número de crianças com necessidades especiais de saúde e apontam que estas vivem marginalizadas e não são reconhecidas pelos sistemas de saúde, educação, transporte, dentre outros.

Nos Estados Unidos, embora as taxas de prevalência não sejam exatas, em um estudo que objetivava realizar uma estimativa nacional do número de crianças com necessidades especiais de saúde e suas características, observou-se que aproximadamente $13 \%$ das crianças do país enquadravam-se neste grupo ${ }^{11}$. O número de crianças dependentes de tecnologia também é desconhecido no Reino Unido, considerando não haver base de dados que retrate esta estimativa; no entanto, as informações disponíveis sugerem que as crianças dependentes de tecnologia têm menos de dois anos de idade, podendo haver variações regionais em relação à prevalência desses $\operatorname{casos}^{12}$.

No Brasil, pesquisadores do Rio de Janeiro desenvolveram um estudo objetivando caracterizar as crianças egressas da terapia intensiva pediátrica e categorizá-las de acordo com os tipos de cuidados demandados após a alta. Como resultado, identificaram que $6,3 \%$ destas crianças foram consideradas com necessidades especiais ${ }^{13}$.

A ausência de dados epidemiológicos representa um desafio para os profissionais de saúde, pois eles se deparam com a invisibilidade dessa clientela nas taxas oficiais e nas políticas públicas, dificultando o planejamento e a implementação de estratégias direcionadas a essas crianças.

Assim, o objetivo é caracterizar as crianças dependentes de tecnologia, residentes no município de Ribeirão Preto - SP, de acordo com as condições de gestação e nascimento, a origem das necessidades especiais de saúde e as demandas de cuidados.

\section{MÉTODO}

Trata-se de um estudo descritivo com delineamento transversal e abordagem quantitativa ${ }^{14}$. A investigação foi realizada no município de Ribeirão Preto, situado na região Nordeste do estado de São Paulo.

Considerando o envolvimento de seres humanos na pesquisa, o estudo foi submetido à apreciação do Comitê de Ética em Pesquisa em cumprimento à Resolução 196/96 da Comissão Nacional de Ética em Pesquisa do Conselho Nacional de Saúde e aprovado sob o número de protocolo 405/ CEPCSE-FMRP-USP, em 10 de agosto de 2010.

Estabeleceram-se, como critérios de inclusão, crianças com até 12 anos de idade, seguindo a determinação do Estatuto da Criança e do Adolescen- 
$\mathrm{te}^{15}$, residentes no município de Ribeirão Preto-SP e dependentes de algum dispositivo tecnológico para manutenção de sua vida.

Considerando que as informações sobre esse grupo de crianças não estavam sistematizadas nos serviços de saúde, foi realizada uma busca ativa pelo município, no período de outubro a dezembro de 2010. Primeiramente foi estabelecido contato com as enfermeiras da rede básica de saúde do município, composta por 47 estabelecimentos, distribuídos em 28 Unidades Básicas de Saúde (UBS), 13 Unidades de Saúde da Familia (USF), cinco Unidades Básicas e Distritais de Saúde (UBDS) e uma Unidade Básica e Especializada; acrescenta-se a esses serviços municipais o Serviço de Assistência Domiciliar - SAD e o Núcleo de Apoio ao Deficiente Físico- NADEF ${ }^{16}$. Explanava-se sobre o projeto e solicitava-se a colaboração na indicação de crianças dependentes de tecnologia daquela área de abrangência, não somente as que eram acompanhadas pelo serviço, mas também aquelas atendidas em outras instituições.

As instituições de apoio às crianças com necessidades especiais de saúde cadastradas junto ao Conselho Municipal dos Direitos da Criança e do Adolescente, conforme especificado nos artigos 90 e 91 do Estatuto da Criança e do Adolescente ${ }^{15}$, também foram visitadas. Dentre as instituições estavam: Associação de Mielomeningocele e Hidrocefalia; Cantinho do Céu- Lar dos Excepcionais; Centro de Atividades Educacionais Especializadas de Ribeirão Preto- CAEERP; Centro Ann Sullivan do Brasil; Núcleo Infantil "Dr. Camilo de Matos"; Hospital de Retaguarda Francisco de Assis; Ambulatório de Assistência Médico Domiciliar- Universidade de Ribeirão Preto e o Centro de Atendimento Odontológico a Pacientes Especiais da Faculdade de Odontologia da Universidade de São Paulo.

Embora essas instituições possuíssem uma lista com os nomes de todas as crianças cadastradas, as informações referentes ao uso de dispositivos nem sempre estavam registradas. Dessa forma, iniciou-se um trabalho de articulação junto aos profissionais envolvidos no cuidado às crianças para esclarecimentos sobre a utilização dos dispositivos tecnológicos. Duas instituições que prestam cuidados a essa clientela no município de Ribeirão Preto não aceitaram participar da pesquisa.

Também foram elaboradas estratégias que visavam divulgar a pesquisa em meios de comunicação escrita e falada. Além disso, foram afixados 150 cartazes pelas unidades de saúde e serviços de apoio convidando as famílias cujas crianças se encontravam em uso de alimentação por sonda, gastrostomia, traqueostomia, oxigenioterapia, ventilação mecânica, em diálise peritoneal, entre outros para participar do estudo. Cabe ressaltar que essas estratégias, embora utilizadas com sucesso em estudos internacionais, não causaram impacto expressivo neste estudo, uma vez que, mesmo com sua utilização, apenas quatro interessados em participar da pesquisa se apresentaram.
Acreditando na possibilidade de haver crianças dependentes de tecnologia que não estavam cadastradas em nenhum serviço público de saúde, foi feito contato com os serviços privados que prestam assistência domiciliária. Em Ribeirão Preto, são dois grandes convênios que atendem à população, sendo que um deles informou que seu atendimento é preferencialmente para adultos e que, raramente, há alguma criança. Foi informado também que, além do acompanhamento médico e internações realizadas por meio do convênio, o Sistema Único de Saúde contribuía com o fornecimento de insumos, como medicação, materiais de uso diário, torpedo de oxigênio e outros equipamentos. Assim, as três crianças acompanhadas neste serviço também eram seguidas pelo Serviço de Assistência Domiciliar da Secretaria Municipal e, portanto, já estavam incluídas no estudo. Com relação ao segundo convênio, houve recusa em participar do estudo.

Para identificar as crianças dependentes de tecnologia contou-se ainda com a estratégia de informantes-chave ${ }^{14}$. Dessa maneira, à medida que era feito contato com uma família, buscava-se saber se conheciam outras crianças na mesma condição. Essa estratégia possibilitou o levantamento de um número expressivo de crianças não cadastradas nos serviços de saúde.

Ao finalizar a busca ativa, identificou-se 124 crianças que atendiam aos critérios de inclusão. As visitas domiciliares ocorreram no período de janeiro a abril de 2011, porém, após a visita, identificou-se que algumas crianças haviam deixado de fazer uso do dispositivo tecnológico e outras tinham falecido. Além disso, foram excluídos alguns casos pela dificuldade de acesso à família, pois estava definido, como critério de exclusão, quatro tentativas de busca sem sucesso. Somente uma mãe se recusou a participar da pesquisa. Assim, ao final, participaram do estudo 102 crianças dependentes de tecnologia, das quais cinco estavam institucionalizadas em instituições de apoio.

$O$ instrumento de coleta de dados aplicado durante as visitas domiciliares foi organizado com dados de identificação da criança, dentre eles endereço e nome do responsável, e questões relacionadas às condições de gestação, nascimento e origem das necessidades. Outra dimensão abordada refere-se a quatro demandas de cuidados: demanda de cuidado medicamentoso, de cuidados de desenvolvimento, de cuidados tecnológicos e demanda de cuidados habituais modificados ${ }^{7}$.

$\mathrm{O}$ instrumento foi preenchido utilizando-se, como fonte, os relatos das mães ou responsáveis; portanto, são dados referidos. Os prontuários dos pacientes foram utilizados somente para preenchimento do instrumento das crianças institucionalizadas, totalizando cinco crianças.

Os dados foram codificados e validados por dupla digitação em planilha formatada do Programa Excel, posteriormente transportados, com vistas à análise descritiva, para o Programa SPSS, versão 16.0. Assim, para as variáveis nominais, os 
resultados serão apresentados sob forma de frequência e porcentagen e, média e desvio padrão, para as numéricas.

\section{RESULTADOS}

\section{Identificação das crianças dependentes de tecnologia}

Da amostra composta por 102 crianças dependentes de tecnologia, 58 (57\%) são do sexo masculino e 44 (43\%) do feminino; idade entre seis meses e 12 anos, principalmente entre um e quatro anos $(40,2 \%)$, média de 5,5 anos (desvio padrão $=3,8)$. Quanto à cor referida pelas mães ou responsáveis, $71(69,6 \%)$ foram consideradas brancas, $21(20,6 \%)$ pardas e $10(9,8 \%)$ negras.

\section{Condições de gestação e nascimento das crianças dependentes de tecnologia}

Na tabela 1 observa-se que, no que se refere à realização do pré-natal, $71(69,6 \%)$ mães compareceram às consultas preconizadas, ou seja, mais de seis consultas; contudo, oito $(7,8 \%)$ não fizeram nenhum acompanhamento nesse período. Quanto ao motivo da não realização do pré-natal, em dois casos foram alegadas dificuldades de agendamento e, em seis casos, por envolvimento da mãe biológica com álcool e drogas ou por problemas psiquiátricos.

As crianças nasceram principalmente de parto cesárea $(63,7 \%)$. Quanto à idade gestacional, a prematuridade ocorreu em $30(29,3 \%)$ dos casos, sendo que destas, oito $(7,8 \%)$ crianças nasceram com menos de 32 semanas de gestação, ou seja, prematuridade extrema.

Em relação ao local de nascimento, observa-se que $98(96,1 \%)$ das crianças nasceram em instituições hospitalares, das quais $38(37,3 \%)$ são oriundas de um hospital de ensino de nível terciário. No total das crianças, apenas três nasceram no domicilio, na unidade básica de saúde e na ambulância.

Em relação aos fatores de risco gestacionais, trata-se de informação referida pelas mães ou responsáveis pelas crianças dependentes de tecnologia como já mencionado. Assim, dos 24 (23,5\%) casos referidos, sete $(29,2 \%)$ tiveram como fator de risco gestacional a gemelaridade; seis (25\%), o uso abusivo de álcool e drogas ilícitas; quatro $(16,7 \%)$ desenvolveram doença hipertensiva específica da gestação; dois $(8,3 \%)$ casos de associação de hipertensão arterial sistêmica e diabetes tipo $2 ; 1(4,2 \%)$ caso de toxoplasmose; um $(4,2 \%)$ caso de diabetes gestacional e um $(4,2 \%)$ de diabetes tipo 1 ; um $(4,2 \%)$ caso de síndrome dos anticorpos antifosfolípideos (SAAF) e um $(4,2 \%)$ caso de ruptura uterina.
Tabela 1: Distribuição das crianças dependentes de tecnologia $(n=102)$ segundo tipo do parto, local de nascimento, idade gestacional, fator de risco gestacional e consultas de pré-natal. Ribeirão Preto - SP, 2011

\begin{tabular}{|c|c|c|}
\hline Variáveis & No & $\%$ \\
\hline \multicolumn{3}{|l|}{ Tipo de parto } \\
\hline Normal & 33 & 32,4 \\
\hline Cesárea & 65 & 63,7 \\
\hline Fórceps & 4 & 3,9 \\
\hline \multicolumn{3}{|l|}{ Local de nascimento } \\
\hline Hospital Filantrópico & 12 & 11,8 \\
\hline Hospital de Ensino & 38 & 37,3 \\
\hline Hospital Privado & 25 & 24,9 \\
\hline Hospital Estadual & 17 & 16,7 \\
\hline Hospital outros municípios & 9 & 8,8 \\
\hline Outros & 3 & 2,9 \\
\hline Não informado & 1 & 1 \\
\hline \multicolumn{3}{|l|}{ Idade gestacional } \\
\hline Prematuro extremo & 8 & 7,8 \\
\hline Prematuro moderado & 10 & 9,8 \\
\hline Prematuro limítrofe & 12 & 11,8 \\
\hline A termo & 71 & 69,6 \\
\hline Não informado & 1 & 1 \\
\hline \multicolumn{3}{|l|}{ Fator de risco gestacional } \\
\hline Sim & 24 & 23,5 \\
\hline Não & 72 & 70,6 \\
\hline Não informado & 6 & 5,9 \\
\hline \multicolumn{3}{|l|}{ Pré- natal } \\
\hline Sim, até 6 consultas & 18 & 17,6 \\
\hline Sim, +6 consultas & 71 & 69,6 \\
\hline Não & 8 & 7,8 \\
\hline Não informado & 5 & 4,9 \\
\hline
\end{tabular}

A média do peso ao nascer foi de 2681 gramas (desvio padrão $=782,9$ ) e a mediana de 2780 gramas, sendo o peso mínimo 635 gramas e máximo 4950 gramas. Quanto à estatura, a média foi de $46,1 \mathrm{~cm}$ (desvio padrão $=5,1$ ) e a mediana de $47 \mathrm{~cm}$, sendo a estatura mínima $28 \mathrm{~cm}$ e máxima $56,5 \mathrm{~cm}$.

Do total de 102 crianças participantes do estudo, os valores do Apgar no primeiro e no quinto minuto estavam disponíveis em 53 (52\%) e 59 $(57,8 \%)$ casos, respectivamente. A não existência dessa informação se deve à falta de registro dos dados na carteira de vacinação da criança. Com relação ao Apgar no primeiro minuto de vida, 36 $(67,9 \%)$ crianças apresentaram valores do Apgar acima ou igual a 7 , indicando ausência de dificuldade de adaptação à vida extra-uterina; 10 (18,9\%) crianças obtiveram valores de Apgar entre 4 e 6, sendo consideradas com asfixia moderada e sete $(13,3 \%)$ crianças com Apgar menor ou igual a 3, classificadas com asfixia grave ao nascer. Com relação ao Apgar no quinto minuto de vida, 47 $(79,6 \%)$ crianças apresentaram valores do Apgar acima ou igual a 7, indicando ausência de dificuldade para adaptação à vida extra-uterina; oito 
(13,6\%) obtiveram valores de Apgar entre 4 e 6, sendo consideradas com asfixia moderada e 4 $(6,8 \%)$ com Apgar menor ou igual a 3 , classificadas com asfixia grave ao nascer.

Origens das necessidades especiais de saúde Quanto às causas adquiridas que ocorreram no período gravídico, no parto e no período neonatal, das 102 crianças, 30 (29,4\%) nasceram prematuras e, destas, $11(36,7 \%)$ necessitaram de internação em unidades de terapia intensiva neonatal; $15(14,7 \%)$ apresentaram o diagnóstico de hipóxia neonatal seguida de internação em unidade de terapia intensiva neonatal e cinco (4,9\%) necessitaram de suporte intensivo logo após o nascimento devido a problema congênito.

As causas congênitas afetaram $67(65,7 \%)$ crianças dependentes de tecnologia, dentre elas estão as malformações do sistema nervoso central, seguidas pelas cardiopatias, malformações crânio-faciais, malformações do sistema digestivo e genito-urinário. Acrescentam-se às causas congênitas algumas síndromes, tais como, Síndrome do Alcoolismo Fetal, Síndrome de Moebius, Síndrome de Dandy-Walker, Síndrome Prunne- Belly, imunodeficiência e amiotrofia espinhal.

Dentre as causas adquiridas não relacionadas ao período gravídico, parto e período neonatal, temos que $31(30,4 \%)$ crianças apresentaram intercorrências ao longo da vida como, por exemplo, neoplasias, afogamentos, queda, mordedura de cão, erro na administração de medicamentos, cirurgias de grande porte e internações em unidades de terapia intensiva pediátrica por agravamento da condição clínica.

As origens das necessidades especiais de saúde, embora neste estudo tenham sido analisadas separadamente, acabam se relacionando umas às outras, podendo haver crianças que tiveram diferentes causas ao longo da vida, provocando o agravamento da condição clínica.

\section{Demandas de cuidado às crianças dependentes de tecnologia}

Quanto à classificação por demanda de cuidados especiais, as 102 (100\%) crianças, pelo objetivo do estudo, apresentam demanda de cuidado tecnológico; 92 (90,2\%) necessitam de cuidado medicamentoso; 74 (72,5\%) demandam cuidado de desenvolvimento e todas requerem cuidados habituais modificados.

Os dispositivos tecnológicos foram divididos em três grupos, aqueles relacionados à alimentação, como sonda nasogástrica, gastrostomia e jejunostomia; os referentes à eliminação urinária ou intestinal, dentre eles a colostomia, ileostomia, sondagem vesical e lavagem intestinal e os dispositivos relacionados à necessidade de oxigenação, como torpedo de oxigênio, concentrador de oxigênio, cateter nasal, traqueostomia e ventilação mecânica.

Das 102 crianças, 71 (69,6\%) utilizam dispositivos tecnológicos para alimentação, sendo que
$61(85,9 \%)$ o fazem por gastrostomia e $10(14,1 \%)$ utilizam sonda nasogástrica ou nasoentérica. Quanto aos dispositivos para eliminação, $29(28,4 \%)$ crianças os utilizam; destas, $17(58,6 \%)$ realizam sondagem vesical de alívio, seis $(20,7 \%)$ têm colostomia ou ileostomia, duas $(6,9 \%)$ necessitam de lavagem intestinal, uma $(3,4 \%)$ possui esofagostomia, duas $2(6,9 \%)$ realizam tanto sondagem vesical de alívio como lavagem intestinal e uma $(3,4 \%)$ mantém ureterostomia. Quanto ao uso de dispositivos para a respiração, das 31 (30,4\%) crianças, $15(48,4 \%)$ têm traqueostomia em ar ambiente; nove (29\%) recebem oxigenioterapia por meio de cateter nasal; três $(9,7 \%)$ permanecem com a traqueostomia e utilizam oxigênio e quatro $(12,9 \%)$ necessitam de ventilação mecânica contínua ou parcial.

A média do tempo de uso dos dispositivos tecnológicos foi de 38,2 meses (desvio padrão $=32,5)$, mediana de 24 meses, sendo o tempo mínimo de um mês e, o máximo, de 144 meses.

Das crianças participantes do estudo, 92 $(90,2 \%)$ apresentam demanda de cuidado medicamentoso. Dentre as medicações de uso diário mais utilizadas pelas crianças dependentes de tecnologia estão os anticonvulsivantes, com 57 $(62 \%)$ crianças em uso; $29(31,5 \%)$ com antibioticoterapia; $28(30,4 \%)$ em uso de medicamentos antirrefluxo; $26(28,3 \%)$ utilizando suplementos alimentares e vitaminas; $21(22,8 \%)$ com corticosteróides e $19(20,7 \%)$ em uso de relaxantes musculares. Ainda em menor frequência, algumas crianças fazem uso de diuréticos, anti-hipertensivos, hormônios e antialérgicos.

Com relação à demanda de cuidado de desenvolvimento, destaca-se a fisioterapia, com 67 $(65,7 \%)$ crianças em acompanhamento, seguida da fonoaudiologia, com 50 (49\%) e a terapia ocupacional, com $21(20,6 \%)$ crianças. Equoterapia e natação surgiram em menor frequência, sendo realizadas por apenas três $(2,9 \%)$ e quatro $(3,9 \%)$ crianças, respectivamente.

As demandas de cuidados habituais modificados compreendem os cuidados do dia a dia que vão além daquele oferecido a uma criança considerada saudável; assim, 62 (60,8 \%) entrevistados afirmaram realizar cuidados específicos para prevenção de novas infecções, dentre eles, esterilização de materiais e alimentos e evitar locais aglomerados, como festas e shoppings. Como uma parcela expressiva de crianças apresenta dificuldades de movimentação, $47(46,1 \%)$ mães ou responsáveis relataram realizar cuidados para a prevenção de úlceras e deformidades. Das 102 crianças participantes do estudo, $45(44,1 \%)$ exigiam cuidados relacionados à prevenção de bronco-aspiração. Monitorização contínua de crises convulsivas e dos sinais vitais e saturação de oxigênio também são cuidados habituais modificados exigidos pelas crianças, correspondendo a $39(38,2 \%)$ e $16(15,7 \%)$, respectivamente. 


\section{DISCUSSÃO}

Os resultados do presente estudo são semeIhantes aos encontrados em outras investigações envolvendo essa clientela, dentre eles destaca-se a predominância do sexo masculino, representando $57 \%$ das crianças participantes ${ }^{11,17}$. O aumento da sobrevida de CRIANES é outro ponto a ser destacado, revelado pela existência de 54,9\% das crianças com idade acima de 5 anos, correspondendo à afirmação de que os avanços e a modernização tecnológica e farmacológica garantem a sobrevivência de muitas crianças ${ }^{11,18}$.

A associação entre a origem da necessidade especial de saúde e as causas perinatais confirma as estatísticas epidemiológicas que indicam a morbimortalidade por afecções perinatais como uma curva ascendente, responsável por mais de $50 \%$ dos óbitos em crianças menores de cinco anos em $2004^{2}$. Essa associação indica a necessidade de desenvolvimento de ações que qualifiquem os serviços de atendimento perinatal, incluindo medidas preventivas de complicações no parto e nascimento ${ }^{2-3}$.

A prematuridade, situação ocorrida com 30\% das crianças dependentes de tecnologia, requer intervenção clínica, farmacológica e nutricional até a criança atingir maturidade para a adaptação à vida extra-uterina, necessitando, na maioria dos casos, de internação em unidades de terapia intensiva neonatal. Dessa forma, esses neonatos egressos da terapia intensiva neonatal tornam-se diferentes dos demais à medida que recebem alta, com inúmeros desafios para realizar o cuidado ${ }^{18}$.

As malformações congênitas enquanto outro fator originário das necessidades especiais de saúde representam a segunda causa de mortalidade nessa faixa etária, chegando, em alguns estados brasileiros, a $30 \%$ dos óbitos em menores de um $a^{19}{ }^{19}$. Nesse sentido, existem estratégias governamentais para sua prevenção a partir de medidas como o uso do ácido fólico antes da concepção e o rastreamento de doenças por meio do Teste do Pezinho ${ }^{20}$. Além disso, os avanços na medicina diagnóstica têm contribuído para que a maioria das malformações possa ser detectada ainda no período gestacional, possibilitando o planejamento antecipado para o nascimento do bebê em uma instituição que ofereça suporte adequado e equipe multiprofissional experiente ${ }^{19}$.

\section{REFERÊNCIAS}

1. Macinko J, Guanais FC, Souza MFM. Evaluation of the impact of the family health program on infant mortality in Brazil, 1990-2002. J Epidemiol Community Health. 2006; 60(1): 13-19.

2. Ministério da Saúde. Saúde Brasil 2004: uma análise da situação de saúde. Brasília: Ministério da Saúde; 2004. 364p.
As causas adquiridas ao longo da vida da criança também são determinantes das necessidades especiais de saúde, como exemplificado neste estudo, por afogamento, mordedura de cão e quedas. Os acidentes e as violências são denominados causas externas e constituem-se em importantes fatores de morbimortalidade infantil e de incapacidades permanentes, ocasionando impacto social, econômico e emocional ${ }^{21}$. Desta forma, é importante o planejamento de ações preventivas e o estabelecimento de estratégias de educação das famílias para uma vigilância efetiva e diminuição de situações traumáticas.

Os achados reafirmam que a transferência de uma criança dependente de tecnologia do hospital para o ambiente domiciliar representa grande desafio, na medida em que essas crianças e seus familiares vivenciam problemas complexos de ordem médica, social e afetiva, os quais não podem ser gerenciados em apenas uma unidade de cuidado ${ }^{-23}$. Uma criança dependente de tecnologia no domicilio requer, dos seus cuidadores, a responsabilidade pelo desempenho de procedimentos altamente técnicos que, formalmente, deveriam ser realizados somente por profissionais qualificados ${ }^{4}$; esses procedimentos incluem troca da cânula de traqueostomia, aspiração de vias aéreas, preparo e administração de medicação, alimentação enteral e higienização de crianças com estomas.

As demandas de cuidados exigidos por essas crianças são especializadas e não se inserem no cotidiano das famílias. Para tanto, a enfermagem pode tornar-se uma aliada a partir do compartilhamento entre seus saberes técnico-científicos e os conhecimentos advindos do saber popular dos cuidadores, contribuindo para o bem-estar da criança, evitando complicações e reinternações frequentes ${ }^{8}$.

A alta planejada conjuntamente com a família e a visita domiciliar de acompanhamento tornam-se uma importante fonte de apoio, por meio de informações, atenção individualizada e seguimento contínuo, minimizando os sentimentos de ansiedade e medo ${ }^{24}$. Nessa perspectiva, faz-se necessário ampliar a visibilidade dessa clientela nas taxas oficiais e nas políticas públicas por meio da ampliação e aprofundamento de estudos desta natureza, os quais podem se constituir em subsídios para pensar o processo de cuidado às crianças com necessidades especiais de saúde e suas famílias.

3. Drumond EF, Machado CJ, França E. Óbitos neonatais precoces: análise de causas múltiplas de morte pelo método Grade of Membership. Cad Saúde Pública. 2007; 23(1): 157-166.

4. Kirk S, Glendinning C, Callery P. Parent or nurse? The experience of being the parent of a technology - dependent child. J Adv Nurs. 2005; 51(5): 456-64. 
5. Brown-Hellsten M. Doença Crônica, Incapacidade ou Tratamento Terminal para a Criança e Família. In: Hockenberry MJ, Wlison D, Winkelstein ML. Wong Fundamentos de Enfermagem Pediátrica. $7^{a}$ ed. Rio de Janeiro (RJ): Guanabara- Koogan, 1999. p. 549-88.

6. McPherson MG, Arango $P$, Fox $H$, Lauver $C$, McManus M, Newachek PW, et al. A new definition of children with special health care needs. Pediatrics. 1998; 102(1): 137-41.

7. Kirk S, Glendinning C. Supporting "expert" parents- professional support and families caring for a child with complex health care needs in the community. Int J Nurs Stud. 2002; 39: 625-35.

8. Neves-Vernier ET. O empoderamento de cuidadoras de crianças com necessidades especiais de saúde: interfaces com o cuidado de enfermagem. [tese]. Rio de Janeiro: Escola de Enfermagem Anna Nery, Universidade Federal do Rio de Janeiro; 2007. p. 172.

9. Leal RJ. Os (des) caminhos dos cuidados às crianças com necessidades especiais de saúde no discurso das famílias: subsídios para uma política de seguimento. [tese]. Rio de Janeiro: Escola de Enfermagem Anna Nery, Universidade Federal do Rio de Janeiro; 2007. p. 141.

10. Vernier ETN, Cabral IE. Caracterização das crianças com necessidades especiais de saúde e seus familiares cuidadores, Santa Maria (RS), 2004-2005: subsídios para intervenções de enfermagem. Rev Soc Bras Enferm Ped. 2006; 6 (1): 37-45.

11. Dyck PC, et al. Prevalence and characteristics of children with special health care needs. Arch Pediatr Adolesc Med. 2004; 158: 885-90.

12. Glendinning C, Kirk S., Guiffrida AE, Lawton D. Techonology- dependent children in community: definitions, numbers and costs. Child Care Health Dev. 2001; 27(4): 321-334.

13. Cabral IE, Silva JJ, Zillmann DO, Moraes JRMM, Rodrigues EC. A criança egressa da terapia intensiva pediátrica na luta pela sobrevida. Rev Bras Enferm. 2004; 57(1): 35-9.

14. Polit D, Beck CT, Hungler B. Delineamentos para a pesquisa em Enfermagem. In: Polit $D$, Beck $C T$, Hungler B. Fundamentos de pesquisa em enfermagem: métodos, avaliação e utilização. $5^{a}$ ed. Porto Alegre (RS): Artmed; 2004. p. 161-244.
15. Brasil. Ministério da Saúde. Estatuto da Criança e do Adolescente. Lei no 8.069 de 13 de julho de 1990. Dispõem sobre a proteção integral da criança e do adolescente. Diário Oficial [da] Republica Federativa do Brasil, Brasília, 16 jul.1990.

16. Ribeirão Preto, 2009. Relatório de Gestão da Secretária Municipal de Saúde do Município de Ribeirão Preto - 2010 (endereço http:// www.ribeiraopreto.sp.gov.br/ssaude).

17. Kirk S. How children and young people construct and negotiate living with medical technology. Social Science \& Medicine. 2010; 71: $1796-1803$.

18. Cabral IE, Moraes JRMM, Santos FF. O egresso da terapia intensiva neonatal de três instituições públicas e a demanda de cuidados especiais. Esc Anna Nery Rev Enferm. 2003; 7 (2): 210-217.

19. Reis AT. O significado da cirurgia neonatal na presença de malformações congênitas: a visão materna para o cuidar de enfermagem. [tese]. Rio de Janeiro: Escola de Enfermagem Anna Nery, Universidade Federal do Rio de Janeiro; 2010.

20. Gaíva MAM, Corrêa ER, Santo EARE. Perfil clínico- epidemiológico de crianças e adolescentes que vivem e convivem com espinha bífida. Rev Bras Crescimento Desenvolvimento Hum. 2011; 21(1): 99-110.

21. Silva MAI, Pan R, Melo L, Bortoli OS, Nascimento LC. Perfil dos atendimentos a crianças e adolescentes vítimas de causas externas de morbimortalidade, 2000-2006. Rev Gaúcha Enferm. 2010; 31(2): 351-8.

22. Fracolli RA, Angelo MA Experiência da família que possui uma criança dependente de tecnologia. Rev Min Enferm. 2006; 10(2): 125-131.

23. Wang KW, Barnard A. Technology-dependent children and their families: a review. J Adv Nurs. $2004 ; 45(1)$ : 36-46.

24. Pinto JP, Ribeiro CA, Pettengill MAM. O processo de recuperação da criança após a alta hospitalar: revisão integrativa. Acta Paul Enferm. 2010; 23(6): 837-42. 